\title{
Merleau-Ponty, l'épistémologie médiationnelle et le mythe du donné
}

Par EMMANUEL CHAPUT

University of Ottawa

Résumé Dans leur critique de l'épistémologie «médiationnelle », Dreyfus et Taylor se revendiquent explicitement de l'héritage merleau-pontien. Leur critique s'articule sur deux fronts, à savoir la critique du représentationalisme et celle du fondationalisme, qui sont les deux éléments constitutifs par excellence de l'épistémologie médiationnelle. Affirmer la valeur d'une position comme celle défendue par Dreyfus, Taylor et Merleau-Ponty ne peut cependant se faire qu'à la condition de bien vouloir la mettre à l'épreuve et en interroger les présupposés. Dans cet article, nous nous demanderons, en retournant aux textes de Merleau-Ponty, si la critique de l'épistémologie médiationnelle ne retombe pas elle-même dans une forme insoupçonnée de fondationalisme. Pour ce faire, nous mettrons Merleau-Ponty à l'épreuve de Sellars et sa critique du donné (Given).

\section{Introduction}

Dans leur critique de l'épistémologie «médiationnelle», Dreyfus et Taylor se revendiquent explicitement de l'héritage merleau-pontien. Leur critique s'articule essentiellement sur deux fronts - à leurs yeux indissociables — à savoir la critique du représentationalisme et celle du fondationalisme qui sont les deux éléments constitutifs par excellence de l'épistémologie médiationnelle ${ }^{1}$. Dans le présent texte, nous défendrons la position critique de

\footnotetext{
${ }^{1}$ On a parfois défendu l'idée que la reprise dreyfussienne de Merleau-Ponty aboutissait, non pas à la critique du fondationalisme médiationnel, mais à la refondation d'un nouveau fondationalisme reposant sur les capacités pré-linguistiques et pré-
} 
Dreyfus et Taylor en retournant aux textes mêmes de Merleau-Ponty pour étayer d'avantage en quoi leur approche s'inscrit dans la même lignée que celui-ci. Merleau-Ponty propose en effet une alternative originale aux tentatives naturaliste et «intellectualiste» de sortie du cadre médiationnel. Affirmer la valeur d'une position comme celle défendue par Dreyfus, Taylor et Merleau-Ponty ne peut cependant se faire qu'à la condition de bien vouloir la mettre à l'épreuve et en interroger les présupposés. Après avoir exposé la nature de la solution merleau-pontienne à l'impasse du médiationalisme, nous nous attarderons donc à voir si celle-ci ne retombe pas dans une nouvelle forme de fondationalisme. En effet, l'être-au-monde de la conscience naïve, à partir duquel Merleau-Ponty entend dériver la conscience réflexive propre à l'objectivité scientifique, se pose originairement dans un rapport immédiat au monde et fonde ainsi la possibilité de la connaissance. Or, l'abandon du cadre médiationnel exige, comme l'affirme avec force Taylor ${ }^{1}$, le rejet de toute forme de fondationalisme. D'où l'importance de déterminer si la solution merleau-pontienne sur laquelle s'appuient Dreyfus

conceptuelles de l'être humain que Dreyfus nomme coping (ou capacité d'adaptation). Voir à ce sujet, J. C. Berendzen, « Coping Without Foundations : On Dreyfus's Use of Merleau-Ponty », International Journal of Philosophical Studies, vol. 18 , no. 5, 2010, p. 629-49. Berendzen rejette un tel fondationalisme se revendiquant de Merleau-Ponty en arguant que ce dernier considère toute expérience humaine comme étant toujours déjà prise dans un réseau de sens culturels qui implique à la fois des éléments linguistiques et conceptuels invalidant par le fait même le fondationalisme phénoménologique de Dreyfus : "Phenomenology of Perception does present the view that all human experience contains cultural aspects that are characteristically marked by linguistic, and thus conceptual, elements. Because of this, the phenomenological foundationalist view is not rightly associated with Merleau-Ponty's work » (ibid., p. 635). Si nous partageons la conclusion de Berendsen, à savoir que Merleau-Ponty n'est pas un fondationaliste, notre argument pour arriver à cette conclusion, sera sensiblement différent, comme nous le verrons plus loin. Par ailleurs, la critique faite par Berendzen de l'interprétation dreyfussienne de Merleau-Ponty semble avoir été rendue caduque par celui-ci. En effet, dans ses écrits ultérieurs, et notamment dans l'ouvrage qui nous intéressera particulièrement ici, Retrieving Realism (2015), coécrit avec Taylor, Dreyfus semble rejeter tout fondationalisme, y compris celui qu'il aurait pu défendre antérieurement en se revendiquant des travaux de Merleau-Ponty. Nous reviendrons plus loin sur la question du fondationalisme, mais il semble bien que l'entreprise de Taylor et Dreyfus vise non pas à « refonder » une position fondationalisme, mais à en faire la critique explicite.

${ }^{1}$ Ch. Taylor, «Foundationalism and the Inner-Outer Distinction », in N. H. Smith (ed.), Reading McDowell on Mind and World, London, Routledge, 2002, p. 109. 
et Taylor revêt implicitement une telle position fondationaliste. Nous mettrons ainsi, dans la troisième section de ce texte, la position merleaupontienne à l'épreuve de la critique sellarsienne du mythe du donné. À la lumière de cette épreuve, nous montrerons en quoi et comment MerleauPonty échappe dans les faits à l'accusation d'être fondationaliste.

Notre texte se divisera ainsi en trois parties. Dans une première partie, nous présenterons le projet critique de Dreyfus et Taylor à la lumière de l'apport merleau-pontien. La seconde partie du texte présentera brièvement la critique merleau-pontienne du naturalisme et son inspiration husserlienne, pour ensuite poser la question de savoir comment la philosophie de MerleauPonty échappe au piège du mythe du donné.

\section{L'impasse des épistémologies post-cartésiennes et sa résolution phénoménologique}

Ce que Dreyfus et Taylor nomment, dans leur ouvrage Retrieving Realism, «l'épistémologie médiationnelle» (mediational epistemology) ${ }^{1}$ trouve ses origines dans la philosophie cartésienne et demeure, selon ceux-ci, le paradigme dominant en épistémologie, bien que l'on ait depuis longtemps cessé de se revendiquer de Descartes ${ }^{2}$. Pour dépasser ce paradigme «médiationaliste » en épistémologie, Dreyfus et Taylor se revendiquent explicitement de Merleau-Ponty.

Traditionnellement, l'épistémologie dite médiationnelle s'est caractérisée par son représentationalisme, mais aussi par son fondationalisme. En fonction de son biais représentationaliste, l'épistémologie médiationnelle situait le problème de la connaissance dans l'articulation entre nos représentations mentales, nos idées, et la réalité du monde. La vérité-correspondance - veritas est adaequatio intellectus et rei - ne peut se réaliser que «par l'entremise ${ }^{3}$ d'une médiation entre l'esprit et le monde, que ce

${ }^{1}$ Cf. H. Dreyfus \& Ch. Taylor, Retriving Realism, Cambridge, Harvard University Press, 2015, p. 2 ; Charles Taylor, «Merleau-Ponty and the Epistemological Picture ", in Taylor Carman \& Mark B. N. Hansen (eds.), The Cambridge Companion to Merleau-Ponty, Cambridge, Cambridge University Press, 2006, p. 26.

2 « Descartes is not in fashion these days. He is rejected as a dualist, as too rationalist, as clinging to an outmoded psychology, and for many other reasons. Yet even though his terms are repudiated, we frequently find the basic structure remaining in place » (Taylor, « Merleau-Ponty and the Epistemological Picture », art. cit., p. 27). Cf. H. Dreyfus \& Ch. Taylor, Retriving Realism, op. cit., p. 3-5.

${ }^{3}$ Ibid., p. 2. 
soit via les simulacres épicuriens ou les idées cartésiennes ${ }^{1}$. Un tel représentationalisme est probablement l'élément le plus caractéristique et le plus durable de l'épistémologie médiationnelle, c'est aussi le plus explicitement pris à partie par les travaux de Merleau-Ponty ${ }^{2}$. Il ne faudrait pas pour autant négliger l'autre dimension caractéristique de l'épistémologie médiationnelle, son fondationalisme.

Le fondationalisme cherche à découvrir un socle imperméable à toute forme de doute, un donné (Given) d'une certitude absolue, à partir duquel nous pourrions reconstruire l'ensemble de notre savoir de manière rigoureuse, claire et distincte ${ }^{3}$. Une telle entreprise fondationnelle peut aussi bien prendre une forme rationaliste qu'empiriste. Le donné peut tout aussi bien être le cogito, la conscience comme substance pensante, à partir duquel on produit le monde objectif - c'est ce que Merleau-Ponty appelle «l'intellectualisme $»^{4}$ - que les sense-data, ces données réelles produisant causalement nos représentations mentales du monde - selon la position « réaliste ».

Dans les deux cas, malgré les divergences, un donné absolument premier sert de base à l'édifice de nos savoirs. Bien entendu, le fondationalisme épistémologique d'un Descartes ou d'un Locke qui constitue la première mouture de l'épistémologie médiationnelle ${ }^{5}$ fut la cible de nombreuses critiques tout au long du vingtième siècle, sans pour autant que l'on sorte réellement du cadre médiationnel. C'est donc dire que le rejet du fondationalisme et du « Mythe du donné » qui y est associé depuis l'ouvrage célèbre de Sellars Empirisme et philosophie de l'esprit ${ }^{6}$ constitue certes une condition nécessaire, mais non suffisante, pour échapper à la théorie média-

\footnotetext{
${ }^{1}$ Maurice Merleau-Ponty, La Structure du comportement [dorénavant SC], Paris, PUF, 2013, p. 289.

${ }^{2}$ Ibid., p. 263.

${ }^{3}$ " The aim of foundationalism is to peel back all the layers of inference and interpretation, and get back to something genuinely prior to them all, a brute Given : then to build back up, checking all the links in the interpretative chain. Foundationalism involves the double move, stripping down to the unchallengeable, and building back up » (Ch. Taylor, « Foundationalism and the Inner-Outer Distinction », art. cit., p. 109).

${ }^{4}$ Elle constitue, selon Merleau-Ponty, une perspective commune à Descartes et Kant qui, à cet égard, sont discutés conjointement. $C f$. SC, p.300 : «Une philosophie d'inspiration criticiste reprend la conception de la connaissance qui a été enseignée par Descartes ».

${ }^{5}$ Ch. Taylor, « Merleau-Ponty and the Epistemological Picture », art. cit., p. 30.

${ }^{6}$ Cf. W. Sellars, Empirisme et philosophie de l'esprit, Paris, L’Éclat, 1992, p. 36, $\S 11$.
} 
tionnelle. Pour Dreyfus et Taylor, c'est seulement lorsqu'on abandonne complètement le cadre représentationaliste qu'il devient possible d'échapper réellement à l'impasse de l'épistémologie médiationnelle ${ }^{1}$.

C'est pourquoi nous nous intéresserons davantage, dans la présente section, à la critique du représentationalisme et à l'alternative proposée par Dreyfus et Taylor, sous l'inspiration de Merleau-Ponty, afin de sortir de l'épistémologie médiationnelle. Il faut toutefois souligner qu'une telle alternative a également la prétention, pour Dreyfus et Taylor, d'échapper au fondationalisme et au mythe du donné. L'analyse de cette prétention fera l'objet de la section 3. Pour le moment, nous nous intéresserons au représentationalisme, aux différentes alternatives qui y sont proposées et en quoi celles-ci, à l'exception de l'approche phénoménologique de Merleau-Ponty reprise par Dreyfus et Taylor, s'avèrent toutes plus ou moins insatisfaisantes.

Selon l'expression de Wittgenstein souvent citée par Taylor : «Une image nous captivait $»^{2}$ (Ein Bild hielt uns gefangen) ; celle du cadre représentationaliste. En effet ce qui caractérise fondamentalement l'épistémologie médiationnelle, selon Dreyfus et Taylor, c'est l'idée selon laquelle la connaissance du monde ne peut passer que par «l'entremise $»^{3}$ (only through, by means of) de nos représentations mentales. L'enjeu et la difficulté de l'épistémologie médiationnelle sont d'être en mesure d'expliquer la correspondance entre le monde des choses et la conscience comme sphère de nos représentations du monde. Entre la réalité objective et notre image subjective de cette réalité, il s'agit de trouver la médiation convenable, le rapport de causalité adéquat, pour établir une vérité-correspondance. Ainsi, toute l'épistémologie post-cartésienne reste animée par le même problème, celui d'établir le rapport de correspondance entre le monde réel et l'esprit.

\footnotetext{
${ }^{1}$ « Now the difference between an antifoundationalism that breaks with the mediational view of knowledge, and one which does not, may seem relatively minor. After all, they both agree in abandonning the original Cartesian enterprise. But in fact, a great deal of the Cartesian philosophical outlook survives if you don't break with the representational epistemology » (H. Dreyfus \& Ch. Taylor, Retriving Realism, op. cit., p. 55).

${ }^{2}$ L. Wittgenstein, Tractatus logico-philosophicus suivi des Investigations philosophiques, Paris, Gallimard, 1986, p. 165, § 115. Cf. Ch. Taylor, « Merleau-Ponty and the Epistemological Picture », art. cit., p. 26 ; « Foundationalism and the Inner-Outer Distinction », art. cit., p. 106 ; H. Dreyfus \& Ch. Taylor, Retriving Realism, op. cit., p. 1.

${ }^{3}$ H. Dreyfus \& Ch. Taylor, Retriving Realism, op. cit., p. 2.
} 
Mais comme on le voit, le point de départ d'une telle problématique, c'est d'emblée la position du «spectateur étranger» ${ }^{1}$, séparé du monde. Selon ce point de vue, il existe un dualisme radical entre la réalité extérieure que l'on cherche à appréhender et les moyens immanents (internes) à la conscience dont on dispose pour réaliser une telle appréhension. C'est ce que Taylor appelle la conception " "Inside/Outside" (I/O) » (Interne/Externe) : il y a, d'une part, le domaine interne de la conscience, de ses représentations et de ses images, et d'autre part, le domaine externe de la réalité, du monde et de la causalité naturelle. Certes, peu nombreux sont ceux qui défendent encore, de façon assumée à la manière de Descartes, un tel dualisme. Et pourtant il semble extrêmement difficile, pour ne pas dire impossible, de sortir d'un tel dualisme, dès lors qu'on l'accepte implicitement comme point de départ de toute réflexion épistémologique. En effet, c'est l'idée même de vouloir sortir de cette conception I/O qui pose problème, dans la mesure où l'on doit alors travailler de l'intérieur cette image du spectateur objectif face au monde pour tenter d'en réconcilier les termes opposés (sujet/objet, interne/externe, etc.). Or, un tel travail semble bel et bien irrémédiablement voué à l'échec. Ce qu'il faut, ce n'est pas sortir de cette image captivante, mais bien l'abandonner ou la recadrer dans un rapport plus originaire au monde.

Comme l'explique Taylor, les tentatives de sortir du dualisme médiationnel I/O consistent le plus souvent à reporter l'un des deux termes en opposition à l'autre ${ }^{3}$. Ce faisant, on n'a fait qu'universaliser l'un des termes du dualisme, soit le monde, soit la conscience, pour ensuite subsumer l'autre pôle du dualisme sous ce terme premier et universel. Le monde est constitué par la conscience transcendantale ou, à l'inverse, la conscience n'est qu'un produit de la causalité physique. Voilà les deux grands programmes qui s'affrontent afin de sortir de l'image médiationnelle en rapportant l'un des deux termes à l'autre. Malheureusement, le plus souvent,

\footnotetext{
${ }^{1}$ SC, p. 245.

${ }^{2}$ Ch. Taylor, «Foundationalism and the Inner-Outer Distinction», art. cit., p. 106. Cf. H. Dreyfus \& Ch. Taylor, Retriving Realism, op. cit., p. 3.

${ }^{3}$ "One "way out" would be to reconstruct the inner zone on the same explanatory principles as disenchanted nature. This is the path of much contemporary cognitive psychology, particularly those strands which have invested deeply in the computer model of the mind. This way of thought is, of course, deeply indebted to the classical dualism, because it has simply taken one of the terms defined by that dualism and universalized it at the expense of the other » (Ch. Taylor, « Foundationalism and the Inner-Outer Distinction », art. cit., p. 107). Cf. H. Dreyfus \& Ch. Taylor, Retriving Realism, op. cit., p. 3.
} 
l'échec ou simplement l'inaboutissement de telles tentatives ouvrent la voie au scepticisme et à un retour au problème I/O. Si l'on ne peut montrer comment la conscience produit le monde ou le monde la conscience, pourquoi ne pas simplement suspendre son jugement et poser la conscience comme séparée d'un monde qu'elle ne peut, au final, pas connaître ? On se retrouve alors à la case départ, face à deux termes séparés qu'on reste incapable de réconcilier.

C'est peut-être alors la stratégie de départ pour sortir du dualisme esprit/monde qui pose problème. Que l'on adopte une approche naturaliste ou matérialiste consistant à ne faire de la conscience qu'une province du monde physique ou, au contraire, que l'on défende plutôt une solution rationaliste, idéaliste ou « intellectualiste » consistant à faire du monde l'expression ou le produit de la conscience, nous nous trouvons devant le même problème. Certes, les apories du réalisme matérialiste ne sont pas celles de l'idéalisme transcendantal, mais le résultat est le même.

La naturalisation de la conscience reste, comme le montre MerleauPonty, un travail non seulement inachevé, mais encore, mal engagé. Les premiers chapitres de La Structure du comportement le montrent bien. Merleau-Ponty y présente une critique du mécanisme des sciences du comportement de son époque. Or, leur volonté de réduire le comportement à un ensemble de réactions à des stimuli physiques simples constitue un projet tout à fait analogue à celui des formes les plus radicales du naturalisme cognitif contemporain ${ }^{1}$. Comme le souligne Merleau-Ponty, de telles approches postulant une sorte de passivité radicale de l'organisme vivant face à son environnement physique tombent facilement dans l'explication unilatérale et abstraite, bien en deçà des faits observés qu'elles tentent d'expliquer :

Puisque tous les mouvements de l'organisme sont toujours conditionnés par des influences externes, on peut bien, si l'on veut, traiter le comportement comme un effet du milieu. Mais de la même façon, comme toutes les

\footnotetext{
${ }^{1}$ C'est d'ailleurs ce que souligne implicitement Dreyfus lorsqu'il oppose la critique merleau-pontienne de l'analyse réelle au courant dominant de la science cognitive contemporaine : "In opposition to mainline cognitive science, which assumes that intelligent behavior must be based on representations in the mind or brain, MerleauPonty holds that the most basic sort of intellignet behavior, skillful coping, can and must be understood without recourse to any type of representation » (H. Dreyfus, "Merleau-Ponty and Recent Cognitive Science », in T. Carman \& M. Hansen (eds.), The Cambridge Companion to Merleau-Ponty, Cambridge, Cambridge University Press, p. 129).
} 
stimulations que l'organisme reçoit n'ont à leur tour été possibles que par ses mouvements précédents, qui ont fini par exposer l'organe récepteur aux influences externes, on pourrait dire aussi que le comportement est la cause première de toutes les stimulations. Ainsi la forme de l'excitant est créée par l'organisme lui-même, par sa manière propre de s'offrir aux actions du dehors ${ }^{1}$.

C'est ce qui fera dire à Merleau-Ponty que le rapport entre l'organisme et son milieu, entre la conscience et le monde ne doit pas simplement être traité de manière unilatérale en rapportant l'un des termes à l'autre, car on perd alors la dimension dialectique du rapport entre le vivant et son milieu, entre l'esprit et le monde. Cette dialectique, qu'elle se situe au niveau physique, vital, symbolique, social ou humain, constitue toujours, pour Merleau-Ponty, « une structure indécomposable $»^{2}$, c'est-à-dire irréductible à l'un ou l'autre des termes qui composent le rapport.

En adoptant systématiquement la perspective de l'analyse réelle partes extra partes, le mécanisme, le naturalisme et le matérialisme s'empêchent de saisir les phénomènes dans leur unité complexe et originaire, et demeurent, pour cette raison, des points de vue abstraits et unilatéraux ${ }^{3}$. C'est pourquoi, pour Merleau-Ponty, le programme naturaliste visant la réduction (naturalisation) de la conscience au monde est mal engagé ${ }^{4}$.

Or, non seulement est-il mal engagé, mais on n'entrevoit toujours pas la possibilité même de son aboutissement. Qu'une telle entreprise puisse réellement être menée à bien, cela tient davantage de la confiance, voire de la foi en la capacité des recherches scientifiques à aboutir à des résultats probants que de la certitude avérée. Certes une telle confiance n'est pas en soi illégitime. Elle s'appuie sur les progrès passés, mais peut-elle cependant résister aux vents et marées de nos échecs et de nos doutes, à l'incertitude à venir? Le propre de la confiance est sa fragilité même, le fait qu'elle puisse toujours se transformer en son contraire. Le scepticisme et l'anti-réalisme risquent alors de réapparaître pour nous couper derechef du monde et de la nature.

\footnotetext{
${ }^{1}$ SC, p. 14.

${ }^{2}$ Ibid., p. 65.

${ }^{3}$ Cf. Th. F. Geraets, Vers une nouvelle philosophie transcendantale - La genèse de la philosophie de Maurice Merleau-Ponty jusqu'à la Phénoménologie de la Perception, La Haye, Martinus Nijhoff, 1971, p. 53.

${ }^{4}$ Nous reviendrons cependant, dans la section suivante, sur les affinités possibles entre Merleau-Ponty et certaines formes moins radicales de naturalisme.
} 
Le même genre de problème hante potentiellement l'intellectualisme que Merleau-Ponty associe principalement au criticisme kantien. À l'inverse du réalisme mécaniste et du naturalisme, le criticisme et plus généralement les philosophies idéalistes entendent reconstituer le monde et la nature à partir de l'activité de la conscience. Une telle approche, dans la mesure où elle vise à réintroduire dans le travail d'ordre scientifique le rôle actif du sujet concevant, semble d'une certaine manière plus proche des positions défendues par Merleau-Ponty lui-même. Il n'en reste pas moins que, pour Merleau-Ponty, la solution kantienne n'a fait que déplacer le problème en ouvrant la porte à un nouveau dualisme non plus entre la conscience et le monde, mais entre les « moi » empiriques et le Je transcendantal :

Partant du spectacle du monde, qui est celui d'une nature ouverte à une pluralité de sujets pensants, elle [la philosophie criticiste de Kant et des postkantiens] recherche la condition qui rend possible ce monde unique offert à plusieurs moi empiriques et la trouve dans un Je transcendantal auquel ils participent sans le diviser [...] C'est pourquoi le problème de la connaissance d'autrui n'est jamais posé dans la philosophie kantienne : le Je transcendantal dont elle parle est aussi bien celui d'autrui que le mien, l'analyse s'est placée d'emblée en dehors de moi, n'a plus qu'à dégager les conditions générales qui rendent possible un monde pour un Je, - moi-même ou autrui aussi bien, et ne rencontre jamais la question : qui médite ? $^{1}$

Certes, pour la philosophie kantienne, le rapport entre les moi empiriques et le Je transcendantal ne pose guère de problème dans la mesure où l'appareil transcendantal du Je, les catégories et les formes de l'intuition, sont partagées de manière nécessaire et universelle par tous. Mais dès que l'on remet en doute cette communauté des moi empiriques en un même Je universel, dès lors qu'on remet en question l'universalité des catégories kantiennes, nous assistons au même retour potentiel du doute sceptique qu'on entrevoyait déjà dans le naturalisme. Nous sommes retournés au point de départ. Si nous mettons de côté la question du statut de la chose en soi chez Kant et postulons que le Je transcendantal est garant de la réalité mondaine, en quoi la conscience du moi empirique est-elle garantie de son adéquation au Je transcendantal?

On comprend dès lors pourquoi toute tentative de sortie de l'épistémologie héritée de Descartes échoue à résoudre la problématique du rapport entre l'esprit et le monde au-delà du doute sceptique. Comme le souligne

${ }^{1}$ M. Merleau-Ponty, Phénoménologie de la perception [dorénavant PP], Paris, Gallimard, 1967, p. 75. 
Taylor, outre ces solutions réductionnistes qui se sont avérées une impasse, une autre manière - inadéquate - d'échapper à l'épistémologie médiationnelle a consisté à simplement se désengager des enjeux d'ordre épistémologique. C'est, aux yeux de Taylor, la position privilégiée par Rorty:

Another popular line, championed by Richard Rorty, is to pour scorn on the hopeless intellectual shifts by which philosophers of the dominant image have unsuccessfully tried to resolve its difficulties, and to call for the abandonment of this whole field of endeavor ${ }^{1}$.

Pour Rorty, l'épistémologie n'est rien d'autre qu'un chapiteau de cirque qui s'est effondré depuis longtemps, mais en dessous duquel les épistémologues cherchent encore, en avançant à tâtons, dans le noir, des solutions à leurs problèmes ${ }^{2}$.

La différence entre Rorty et Taylor, c'est que ce dernier, qui lui aussi, dans la mesure où il prétend échapper à l'image captivante de l'épistémologie post-cartésienne, observerait d'un point de vue extérieur l'effondrement du chapiteau, se refuse néanmoins à l'abandon pur et simple de tout questionnement d'ordre épistémologique : «We think that these [epistemological] questions and distinctions have to be recast $»^{3}$. S'il faut effectivement se libérer du chapiteau effondré, il n'en reste pas moins que le spectacle qui avait cours, bien qu'il fut mal monté, avait néanmoins sa raison d'être. Comprendre la nature de la vérité, le rapport entre esprit et monde, fait partie de ces questions qui fascinent la curiosité intellectuelle de l'être humain à l'instar des questions métaphysiques qui ne cesseront jamais, selon Kant, de nous interpeller. Il faut donc refonder l'épistémologie sur une nouvelle base et non simplement l'abandonner, comme le suggère Rorty. Avec celui-ci, le bébé est jeté avec l'eau du bain. Et c'est pourquoi c'est plutôt du côté de Merleau-Ponty qu'il faut chercher, avec Dreyfus et Taylor, une troisième voie entre les écueils réaliste et intellectualiste.

Plutôt que de travailler l'image représentationaliste de l'intérieur afin de subsumer l'un des termes du dualisme sous l'autre, cette troisième voie cherchera à montrer qu'une telle image qui pose d'emblée une séparation

${ }^{1}$ Ch. Taylor, «Foundationalism and the Inner-Outer Distinction», art. cit., p. 107. Cf. H. Dreyfus \& Ch. Taylor, Retriving Realism, op. cit., p. 41.

${ }_{2}^{2}$ R. Rorty, "Charles Taylor on Truth", in Truth and Progress - Philosophical Papers, vol. 3, Cambridge, Cambridge University Press, 1998, p. 93.

${ }^{3}$ H. Dreyfus \& Ch. Taylor, Retriving Realism, op. cit., p. 41. 
entre le sujet de la connaissance, le " spectateur objectif de la science ${ }^{1}{ }^{\text {et }}$ l'objet de la connaissance, c'est-à-dire le monde, n'a rien d'originaire ${ }^{2}$. Cette dualité entre l'esprit et le monde est un produit dérivé, une image du monde qui n'émerge qu'à partir d'un rapport plus originaire au monde et que Merleau-Ponty nommera l'être-au-monde. Il s'agit alors, pour Merleau-Ponty

de nous présenter, non pas, comme les philosophies classiques, l'esprit et le monde, chaque conscience et les autres, mais la conscience jetée dans le monde, soumise au regard des autres et apprenant d'eux ce qu'elle est. Une bonne part de la philosophie phénoménologique ou existentielle consiste [...] à faire voir le lien du sujet et du monde, du sujet et des autres, au lieu de l'expliquer, comme le faisaient les classiques ${ }^{3}$.

Notre rapport au monde n'est pas d'abord et avant tout celui d'un spectateur, mais bien celui d'un participant. Nous habitons d'abord le monde selon le mode de la familiarité et de l'encastrement (inbeddedness). C'est ce que Dreyfus et Taylor appellent, par opposition à l'approche médiationnelle, la théorie du contact avec le monde (contact theory) :

Where mediation theory seeks knowledge as arising through some mediational element, so that we have contact with the real in knowledge only through some intermediary, depiction, or category, contact theories give an account of knowledge as our attaining unmediated contact with the reality known ${ }^{4}$.

Tant que l'on demeure dans ce que Taylor appelle la vision désenchantée (disenchented $)^{5}$ de la nature consistant à se représenter le monde en termes géométriques et physiques, en termes d'éléments simples et atomiques, une

\footnotetext{
${ }^{1}$ " The aim of "scientific naturalism," as Jennifer Hornsby describes it, is to account for the actions, feelings, intentions, etc., of persons from the "objective, thirdpersonal perspective" that natural scientists adopt» (H. Dreyfus \& Ch. Taylor, Retriving Realism, op. cit., p. 15, n. 16).

${ }^{2}$ «Ce n'est pas avec des "représentations" ou avec une pensée que je communique d'abord, mais avec un sujet parlant, avec un certain style d'être et avec le "monde" qu'il vise » (PP, p. 214).

${ }^{3}$ M. Merleau-Ponty, "Le Cinéma et la nouvelle psychologie », dans Sens et nonsens, Paris, Nagel, 1965, p. 104-05.

${ }^{4}$ H. Dreyfus \& Ch. Taylor, Retriving Realism, op. cit., p. 17.

${ }^{5}$ Ch. Taylor, « Foundationalism and the Inner-Outer Distinction », art. cit., p. 106.
} 
telle contact theory apparaît certainement «naïve $»^{1}$. La science le montre, l'apparence du monde n'est pas garante de la réalité : le Soleil semble mobile et d'une circonférence d'un pied environ, alors qu'on sait qu'il n'en est rien. Comment alors songer sérieusement à remplacer l'épistémologie médiationnelle, certes problématique, mais suffisamment sophistiquée pour rendre compte de notre compréhension scientifique du monde, par une conception naïve de notre être-au-monde qui s'effondre devant la moindre illusion d'optique?

L'approche de Merleau-Ponty consiste cependant non pas à un retour pur et simple à la conscience naïve comme l'horizon ultime de notre savoir. Comme l'écrit Geraets : «Il faut [...] maintenir les deux perspectives dans ce qu'elles ont de légitime, et tenter de comprendre l'homme en cherchant à comprendre leur rapport ${ }^{2} »$. Comprendre un tel rapport n'exclut pas cepen-

\footnotetext{
${ }^{1}$ H. Dreyfus \& Ch. Taylor, Retriving Realism, op. cit., p. 17. Merleau-Ponty dira de même : «L'analyse scientifique du comportement [mais plus généralement, tout analyse scientifique, dans la mesure où elle suppose la position d'un observateur objectif - E.C.] s'est définie d'abord par opposition aux données de la conscience naïve » (SC, p. 5) que Merleau-Ponty décrit, au dernier chapitre de La Structure du comportement, comme une conscience en contact avec son monde. «[B]ien que la conscience naïve ne confonde jamais la chose avec la manière qu'elle a de nous apparaître, écrit Merleau-Ponty, et justement parce qu'elle ne fait pas cette confusion, c'est la chose même qu'elle pense atteindre, et non quelque double interne, quelque reproduction subjective » (SC, p. 283). La conscience naïve est ainsi d'emblée non représentationaliste ; ce qui apparaît, c'est la chose même et non sa reproduction imaginaire. Le réalisme primitif de la conscience naïve est d'emblée une (proto)théorie « contactiste ».

${ }^{2}$ Th. Geraets, Vers une nouvelle philosophie transcendantale, op. cit., p. 35. Nous pourrions être tentés d'inscrire une telle tentative d'articuler les perspectives de la conscience incarnée dans son monde et de l'observateur objectif de la science dans la problématique sellarsienne de l'articulation entre l'image manifeste et scientifique du monde (Cf. W. Sellars, "La Philosophie et l'image scientifique de l'homme », dans D. Fisette et P. Poirier (éds.), Philosophie de l'esprit, t. 1: Psychologie du sens commun et sciences de l'esprit, Paris, Vrin, 2002, p. 81). Il n'est pas certain cependant que les solutions merleau-pontienne ou sellarsienne soient, en définitive, réconciliables. Premièrement, parce que pour Sellars l'articulation de ces deux images se présente nécessairement sous la forme d'une rivalité (ibid., p. 85) et, deuxièmement, parce qu'il semble en définitive accorder un primat à l'image scientifique (ibid., p. 103-11), alors qu'au contraire, Merleau-Ponty fait le pari d'une harmonie possible entre les images manifeste et scientifique en accordant davantage un primat à l'image manifeste de l'être-dans-le monde à partir duquel l'image scientifique émergerait sans jamais pouvoir l'éclipser complètement.
} 
dant que Merleau-Ponty établisse un ordre de priorité entre ces deux perspectives. Il y a, pour Merleau-Ponty, primauté de notre appartenance au monde par rapport à la possibilité d'adopter une perspective détachée face aux choses du monde: «Chacune des équations de la physique présuppose notre expérience préscientifique du monde et cette référence au monde vécu contribue à en constituer la signification valable ${ }^{1}$. Cela ne veut pas dire pour autant qu'une telle position de "spectateur étranger» soit illégitime, elle est simplement dérivée, seconde par rapport à notre être-au-monde.

De manière originaire, le monde ne se décompose pas en éléments simples, mais se présente toujours, au contraire, sous la forme de structures irréductibles, indécomposables. C'est pourquoi, aux yeux de Merleau-Ponty, les sciences du comportement qui tentent d'analyser les réactions animales à des stimuli simples errent complètement. Elles transposent artificiellement à l'animal une perspective proprement humaine, à savoir la vision scientifique du monde décomposant le monde en éléments simples, selon des rapports mathématiques ${ }^{2}$. Au contraire, l'animal perçoit d'abord et avant tout, non pas des éléments simples et disparates qu'il s'agirait de synthétiser, mais des rapports, des structures complexes ${ }^{3}$ que l'être humain seul, par sa capacité de s'élever au-dessus de son être-immédiat, au-monde, et de prendre une distance critique, est en mesure d'analyser, c.-à-d. décomposer, en termes simples. Les poules de Koehler ne réagissent pas à un tas de graines colorées plutôt qu'à un autre, mais bien à un rapport entre des nuances claires ou sombres ${ }^{4}$. "Corrélativement, comme l'écrit Merleau-Ponty, la réaction motrice acquise n'est pas une somme de mouvements individuels », mais le produit d'une "structure afférente [consistant à discriminer systématiquement entre les nuances les plus sombres] qui déclenche et règle une structure motrice $»^{5}$. Autant l'animal que l'être humain saisissent ainsi d'abord et avant tout le monde en termes de structures indécomposables. Ce n'est que

\footnotetext{
${ }^{1}$ PP, p. 494.

2 « Il y a quelque chose d'artificiel dans les descriptions du comportement que nous avons données jusqu'ici; en sollicitant de l'animal des réactions aux relations géométriques et physiques, on donne à croire qu'elles sont le cadre naturel de son comportement comme elles sont, pour l'homme, constitutives du monde » (SC, p. 172-73). Cf. Th. Geraets, Vers une nouvelle philosophie transcendantale, op. cit., p. 43.

${ }^{3}$ « De là vient que l'araignée, si l'on met une mouche dans son nid, ne la traite pas comme une proie. Son comportement instinctif n'est pas une réaction à l'égard de la mouche, mais une réaction à l'égard d'un objet vibrant en général » (SC, p. 149).

${ }^{4}$ SC, p. $161-62$

${ }^{5}$ Ibid., p. 162.
} 
dans un deuxième temps que l'être humain est à même de décomposer ces structures en éléments simples, mais une telle décomposition n'est pas nécessairement pour Merleau-Ponty une appréhension plus adéquate de la structure de la réalité ${ }^{1}$. C'est certainement un complément important à notre compréhension du monde qui jaillit autrement de notre rapport immédiat naï - au monde, mais cela n'épuise pas pour autant tous les sens du monde. Comme l'affirment Dreyfus et Taylor, il est certain que le savoir scientifique, dans la mesure où il est avéré, fournit une propriété essentielle et universellement valide de son objet, mais cela ne veut pas pour autant dire que cette propriété essentielle épuise l'essence, voire les essences possibles d'une chose :

Of course, our scientific understanding, if true, would be true in the world of the Egyptians even though they couldn't understand it; but it doesn't follow that what they meant by gold is determined by our science [...] Understanding gold as essentially having an atomic number of 79 does explain, or at least promises to explain, the causal properties of gold [...] its atomic number may well correspond to the structure of gold as it is in itself. Still, having an atomic number of 79 need not be considered to be the essential property of gold. It is essential only relative to our way of questioning nature so as to reveal its independent properties. The Egyptians might well have revealed properties of gold only accessible through their religious practices ${ }^{2}$.

La stratégie de Dreyfus et Taylor est ainsi de défendre un réalisme pluraliste selon lequel il existe plusieurs façons d'interroger le monde qui, néanmoins, chacune à leur manière, propose un ensemble de vérités sur le monde qui ne sont pas simplement le produit d'une projection de la conscience sur le monde. Autrement dit, la réalité n'est pas qu'une construction, même si elle ne peut être en aucun cas réduite à un seul type d'explication d'ordre causal ${ }^{3}$.

\footnotetext{
${ }^{1} \mathrm{Au}$ contraire, Merleau-Ponty adhère plutôt à un holisme selon lequel la réalité du tout excède ce qu'en révèle la somme de ses parties $(C f$. SC, p. 29; PP, p. 16; Vincent Descombes, Le Même et l'autre, Paris, Minuit, 1979, p. 74).

${ }^{2}$ H. Dreyfus \& Ch. Taylor, Retriving Realism, op. cit., p. 150-51.

${ }^{3}$ C'est ainsi que Dreyfus et Taylor définissent leur réalisme pluraliste robuste (pluralistic robust realism) : "There may be (1) multiple ways of interrogating reality (that's the "plural" part), which nevertheless (2) reveal truths independent of us, that is, truths that require us to revise and adjust our thinking to grasp them (and that's the robust realist part), and where (3) all attempts fail to bring the different ways of interrogating reality into a single mode of questioning that yields a unified picture or theory (so they stay plural) » (ibid., p. 154).
} 
De manière analogue, Merleau-Ponty propose dans La Structure du comportement d'articuler notre savoir selon trois ordres (physique, vital, humain), chacun possédant ses propres structures, ses propres dialectiques, sans que l'on puisse réduire la vérité à l'un ou l'autre de ces trois ordres. « [L]a philosophie de la structure », proposée par Merleau-Ponty vise ainsi à maintenir « le caractère original des trois ordres et admet que quantité, ordre et signification, sont présents dans tout l'univers des formes, sont cependant des caractères "dominants" respectivement dans la matière, la vie et l'esprit $\gg{ }^{1}$. On peut certes penser le corps à partir de l'ordre physique « comme une masse matérielle et inerte $»^{2}$, on n'en saisira pas la dimension vitale. Comme l'écrit Geraets : "Merleau-Ponty insiste sur l'impossibilité de réduire les rapports entre l'animal et son milieu à ceux qu'on trouve dans le monde physique $»^{3}$. S'il n'est pas faux de vouloir traiter le corps comme un composé d'atomes, de tissus cellulaires, à rester sur ce plan (physique) de la réalité, nous passerions à côté de la dimension vivante ou symbolique du corps. Il faut donc, au contraire, chercher à saisir la totalité d'un phénomène à partir des multiples structures et dialectiques qui le pénètrent ${ }^{4}$.

Ainsi, pour Merleau-Ponty, même lorsque nous entreprenons l'analyse des parties, même lorsque nous cherchons à dépasser l'évidence du réalisme naïf pour fonder scientifiquement notre compréhension du monde, notre rapport originaire au monde doit néanmoins être maintenu. Même si cet arrière-plan indépassable qu'est notre être-au-monde est souvent dans l'ombre de certaines attitudes (scientifique, philosophique, phénoménologique, etc.) plus sophistiquées, il reste néanmoins actif: "L' "être-à-lavérité" n'est pas distinct de l'être au monde ${ }^{5}$. Notre être-au-monde consti-

\footnotetext{
${ }^{1}$ SC, p. 200.

${ }^{2}$ Ibid., p. 286.

${ }^{3}$ Th. Geraets, Vers une nouvelle philosophie transcendantale, op. cit., p. 40-41. Conséquemment: "Il faut, au lieu de donner le primat ontologique au monde physique, reconnaître l'égalité de droit entre les trois sortes de formes [physique, vitale, symbolique], c'est-à-dire nier entre elles tout rapport de causalité » (ibid., p. 65).

${ }^{4}$ Autant que le réductionnisme, Merleau-Ponty cherche à dépasser toute vision fragmentaire de l'expérience : «À partir du moment où l'on se refuse à séparer la relation et les différentes structures concrètes qui paraissent dans l'expérience, il n'est plus possible de fonder toute relation sur l'activité du "sujet épistémologique", et, en même temps que le monde perçu se fragmente en "régions" discontinues, la conscience se scinde en actes de conscience de différents types » (SC, p. 260-61). ${ }^{5}$ PP, p. 452.
} 
tue toujours la condition première de notre savoir ${ }^{1}$. Les formes de savoirs les plus détaché de notre appartenance au monde comme corps perceptif, les mathématiques par exemple, existent toujours en définitive, pour MerleauPonty, selon le mode d'une distanciation qui n'est concevable qu'à partir de ce avec quoi on prend ses distances, à savoir notre être-au-monde comme structure indécomposable.

Prenons l'exemple du rapport entre perception et sensation. La perception constitue la «structure » ${ }^{2}$ par excellence par laquelle s'établit notre rapport au monde ${ }^{3}$. Pour Merleau-Ponty le monde se donne à la conscience naïve par le biais de la perception : "Les "choses" dans l'expérience naïve sont évidentes comme êtres perceptifs ${ }^{4}$. Faisant «du corps le sujet de la perception $\rangle^{5}$, Merleau-Ponty pose d'emblée la conscience naïve comme incarnée dans un rapport au monde par le biais de sa structure perceptive. La perception qui, pour Merleau-Ponty, part toujours d'une perspective, celle de notre corps occupant une certaine position dans le monde - position qui en détermine le point de vue et donc le champ perceptif - , constitue la structure indécomposable de notre être-au-monde. Nous sommes à la fois, comme corps phénoménal, dans un monde et, comme corps percevant, regard sur ce monde. Mais un tel regard-sur prend toujours place dans le monde.

À l'opposé, les théories classiques de la perception, partant de la position du spectateur étranger pour lequel le regard-sur est hors de ce qu'il

\footnotetext{
${ }^{1}$ Cf. V. Descombes, Le Même et l'autre, op. cit., p. 77, n. 11. Descombes suggère toutefois la faillite d'une telle tentative de refonder la vérité objective de la science sur la sphère du vécu. Il semble cependant appuyer sa position sur une identification un peu trop rapide à notre avis de la phénoménologie existentielle de Merleau-Ponty avec ses sources husserliennes. Si, de fait, Merleau-Ponty s'inspire largement de Husserl, il n'en demeure pas moins qu'il faut distinguer entre ce qu'il reprend et ce par quoi il innove. Répondre à Descombes demanderait cependant d'élaborer ces points communs et ces points de distinctions existants entre Merleau-Ponty et Husserl, afin de montrer que «l'échec» (aux yeux de Descombes) de la phénoménologie dans sa forme husserlienne n'implique pas de facto l'échec du projet merleaupontien. Cela excède cependant notre propos. Quoi qu'il en soit, Descombes présente, à notre avis, à merveille ce qui constitue le cœur du projet épistémologique de Merleau-Ponty, à savoir, expliciter la légitimité de la perspective scientifiqueobjectiviste à partir de la structure originaire de la conscience.

${ }^{2}$ PP, p. 10.

${ }^{3}$ Cf. ibid., p. 24.

${ }^{4}$ SC, p. 284.

${ }^{5}$ PP, p. 260.
} 
regarde, présentent la perception le plus souvent comme le simple résultat d'une synthèse de sensations. J'ai la sensation du rouge, d'une sphère, etc., j'en déduis que je perçois une pomme. La perception n'a donc rien d'originaire, elle est le produit, le composé d'éléments simples que l'on nomme sensations. Merleau-Ponty renverse complètement cette perspective. Certes, on peut décomposer la perception en une série de sensations, mais seulement après avoir déjà perçu de manière totalisante. $\mathrm{Il}$ faut alors prendre une autre posture phénoménologique, en mettant l'accent sur tel ou tel élément du champ perçu.

À tout moment cependant, ce champ reste la condition de possibilité de mon entreprise de décomposition. Lorsque je me concentre sur un tableau, je continue à le percevoir encadré et accroché au mur, je peux même concentrer mon attention sur un détail du tableau, mais seulement après l'avoir perçu en tant que tableau. Il n'est pas la somme de ses détails. Ses détails sont le fruit d'un réalignement perceptif partant d'une saisie initiale du tout comme unité. C'est ainsi que Merleau-Ponty écrit, dans Phénoménologie de la perception :

C'est à partir du lié que j'ai secondairement conscience d'une activité de liaison, lorsque, prenant l'attitude analytique, je décompose la perception en qualités et en sensations et que, pour rejoindre à partir d'elles l'objet où je m'étais d'abord jeté, je suis obligé de supposer un acte de synthèse qui n'est que la contre-partie de mon analyse. Mon acte de perception, pris dans sa naïveté, n'effectue pas lui-même cette synthèse, il profite d'un travail déjà fait, d'une synthèse générale constituée une fois pour toutes...

Or, c'est seulement à partir du moment où je prends pour point de départ vrai et premier ce résultat dérivé — « produit tardif de la pensée ${ }^{2}$ — de mon analyse distanciée que les dangers de l'épistémologie médiationnelle réapparaissent. Quel est le statut de ces sense-data que je perçois, comment s'opère donc la synthèse perceptive à partir d'une telle multiplicité ? À vouloir expliquer la perception comme le résultat d'une synthèse plutôt que la condition indécomposable de toute analyse en termes de sensations, à négliger l'originarité de la perception comme arrière-plan, comme horizon à partir duquel nous saisissons les éléments simples au prix d'une modification de notre attention et de notre regard, la théorie classique de la perception ouvre la boîte de Pandore médiationnelle. Comme l'écrivait Augustin à propos du temps : «N'y a-t-il personne pour me poser la question, je sais ;

${ }^{1}$ PP, p. 275.

${ }^{2}$ Ibid., p. 17. 
que, sur une question, je veuille l'expliquer, je ne sais plus ${ }^{1}$. En questionnant l'origine de l'originaire en termes d'analyse réelle, en termes de sensations, les théories classiques de la perception cherchent à rendre compte de l'évidence de la conscience naïve au-monde par ce qui est « le moins clair $»^{2}$. La conscience naïve appréhende le monde comme un acteur incarné sans douter que l'ensemble constitutif de son horizon perceptif forme un tout cohérent. Sans se questionner, elle sait. Mais lorsqu'il s'agit d'expliquer cette familiarité, les théories classiques font le pari d'expliquer la perception en la décomposant, en la décortiquant. D'un côté existe ce qui est perçu et qui constitue un ensemble de données simples, de l'autre côté il y a une conscience percevante qui synthétise les données pour en faire des représentations mentales. Dès lors, l'impasse de l'épistémologie médiationnelle réapparaît : comment expliquer la synthèse des sensations susceptibles de produire en moi une représentation adéquate du monde?

Au contraire, en montrant que la possibilité d'une telle décomposition de la perception en éléments simples n'est pas première, mais seulement le fruit d'un déplacement de mon attention susceptible d'être analysé phénoménologiquement, Merleau-Ponty montre comment l'analyse réelle, en termes d'éléments simples, partes extra partes, n'est possible que si l'on maintient en arrière-plan l'unité originaire de notre être-au-monde.

Ainsi, non seulement la conscience naïve est d'une certaine manière supérieure à la conscience philosophico-scientifique empêtrée dans l'image médiationnelle - dans la mesure où pour la conscience naïve, le rapport de notre être-au-monde est foncièrement non problématique ${ }^{3}-$, elle lui serait aussi supérieure par sa capacité à rendre compte des conditions de possibilité de l'image médiationnelle, du regard «scientifique». L'inverse n'est toutefois pas vrai. Pour l'épistémologie médiationnelle, la conscience naïve n'est qu'une conception " primitive » pétrie d'erreur, et ce, bien que nous vivions la grande majorité du temps conformément à cette conception «naïve » du monde ${ }^{4}$.

Nous vivrions ainsi, le plus souvent, dans l'erreur sans que les approches médiationnelles soient en mesure d'expliquer pourquoi une telle

\footnotetext{
${ }^{1}$ Augustin, Confessions, Paris, Pierre Horay, 1961, p. 333. Cf. PP, p. 17.

${ }^{2}$ PP, p. 17.

3 "Les "simulacres" épicuriens ou les "espèces intentionnelles", "toutes ces petites images voltigeantes par l'air" qui apportent dans le corps l'aspect sensible des choses, ne font que transposer en termes d'explications causale et d'opérations réelles la présence idéale de la chose au sujet percevant qui, nous l'avons vu, est une évidence pour la conscience naïve » (SC, p. 289).

${ }^{4}$ Cf. H. Dreyfus \& Ch. Taylor, Retriving Realism, op. cit., p. 37.
} 
« erreur » reste si prenante. La théorie médiationnelle ne peut que rejeter le réalisme naïf de l'être-au-monde comme une conception distordue du monde. Pour Merleau-Ponty au contraire, les deux images, naïve et scientifique, du monde peuvent être maintenues conjointement dans la mesure où l'entreprise critico-analytique de la science peut être dérivée phénoménologiquement de notre être-au-monde comme une forme de distanciation par le réalignement de notre regard sur le monde, par l'adoption d'une posture, celle du spectateur objectif ${ }^{1}$.

Si l'épistémologue médiationaliste rappelle d'une certaine manière l'enfant qui demande constamment à ses parents le pourquoi des choses en cherchant à expliquer la perception par le biais de la sensation ${ }^{2}$, c'est une autre figure de l'enfance - qui saisit la structure d'un visage familier et bienveillant bien avant d'en comprendre les composantes - qui exprime le

\footnotetext{
${ }^{1}$ Dans Titres et Travaux, « un texte inédit que Merleau-Ponty a envoyé en 1952, à tous les professeurs du Collège de France avant la visite qu'il devait leur faire en vue de son élection» (Th. Geraets, Vers une nouvelle philosophie transcendantale, op. cit., p. 32), ce dernier écrit : « Même pour construire un savoir positif, il faut que je m'assure d'avoir accès à mes propres pensées, et d'en pouvoir apprécier la validité intrinsèque. Si je ne suis qu'un produit du milieu et de l'histoire, j'assiste au déroulement de mes événements, sans être capable d'en discerner le sens ni de distinguer en moi le vrai du faux » (cité dans ibid., p. 34). On voit bien là en quoi tout travail d'ordre positif et scientifique suppose, pour Merleau-Ponty, une entreprise préable d'explicitation de leur possibilité à partir de la conscience incarnée, au-monde.

${ }^{2}$ Robert Brandom par exemple, dans « Knowledge and the Social Articulation of the Space of Reasons », définit le savoir au sein de l'espace des raisons comme le jeu consistant à donner et à demander des raisons et des justification, le type de justifications que l'on donnera ou exigera dépendant de notre statut dans cet espace social des raisons : « Knowledge is intelligible as a standing in the space of reasons, because insofar as it is intelligible as a status one can be taken to achieve in the game of giving and asking for reasons » (Robert Brandom, « Knowledge and the Social Articulation of the Space of Reasons ", in E. Sosa \& J. Kim (eds.), Epistemology An Anthology, London, Blackwell, 2005, p. 429). C'est une telle conception du savoir comme étant un jeu où les participant se donnent et se demandent constamment les raisons des choses (the game of giving and asking for reasons) qui inspire cette analogie de l'enfant - qui dans une certaine mesure n'aurait compris que la moitié des règles du jeu — qui demande constamment la raisons des choses à ses parents sans jamais se lasser de demander «pourquoi ». Le monde a alors cessé d'aller de soi et on cherche, à l'instar du scientifique, à en saisir le raison indépendamment de ce qui, pour la conscience naïve, relève de l'évidence.
} 
mieux, pour Merleau-Ponty, l'originarité de notre être-au-monde ${ }^{1}$. Avant d'habiter l'espace des raisons et du langage où l'on prend une certaine distance avec les choses pour chercher le pourquoi et la justification, l'enfant habite d'abord un monde perçu et familier ${ }^{2}$. Il s'agit alors, pour MerleauPonty, d'expliciter (phénoménologiquement) comment une telle prise de distance face au monde (permettant l'attitude scientifique) s'appuie en premier lieu sur un changement de l'attention au sein du sujet percevant (sans pour autant que cela signifie une sortie du champ perceptif).

Ainsi, plutôt que de partir de l'image médiationnelle d'un sujet séparé du monde, la solution inspirée par Merleau-Ponty consiste à poser l'être-aumonde de la conscience naïve comme rapport originaire au monde à partir duquel seul peut émerger le rapport du "spectateur étranger», neutre et détaché du monde. Il s'agit donc d'éclairer les conditions de possibilités de l'attitude scientifique à partir de notre être-au-monde ${ }^{3}$. Une telle solution s'inscrit dans la lignée des critiques phénoménologiques du naturalisme.

\footnotetext{
${ }^{1}$ SC, p. 237. C'est aussi à ce type de données empiriques sur l'apprentissage des enfants et la structure de leur rapport-naissant-au-monde que se réfèrent encore aujourd'hui les défenseurs de la position merleau-pontienne $(C f$. H. Dreyfus \& Ch. Taylor, Retriving Realism, op. cit., p. 36 ; G. Soffer, « Revisiting the Myth : Husserl and Sellars on the Given », The Review of Metaphysics, vol. 57, no. 2, 2003, p. 32325). La question demeure toutefois de savoir si l'originarité de l'être-au-monde, sa primauté sur le regard spectateur du scientifique qui consiste à saisir d'abord le monde en termes de structures plutôt que d'éléments simples n'est prioritaire que d'un point de vue ontogénétique ou si la prétention de Merleau-Ponty est d'y voir véritablement une priorité ontologique et épistémique : le monde est constituée de structures avant d'être fait d'éléments. Du point de vue de l'image scientifique, si l'on peut être prêt à admettre que l'enfant saisit d'abord le complexe structural, on peut néanmoins rejeter l'idée qu'ontologiquement le monde ne soit pas d'abord composé d'éléments simples, atomiques. De ce point de vue, notre accès à la réalité ontologique passe certes par le biais d'une appréhension complexe (première pournous), mais la réalité d'un monde est d'abord le résultat d'une synthèse à partir d'éléments simples constitutifs du monde physique (premiers en-soi).

${ }^{2}$ C'est pourquoi, pour Merleau-Ponty, le langage est à la fois principe d'esclavage et de liberté (SC, p. 263, n. 1). En permettant de dépasser l'adhésion immédiate au milieu pour entretenir un rapport «spectaculaire » au monde — où les choses du monde se déroulent devant moi comme quelque chose d'extérieur - , le langage permet un nouveau regard (scientifique) sur le monde, il nous coupe cependant de notre familiarité originaire avec le monde que l'on habite. $C f$. Th. Geraets, Vers une nouvelle philosophie transcendantale, op. cit., p. 47.

${ }^{3}$ PP, p. 494.
} 


\section{Merleau-Ponty et la critique phénoménologique du naturalisme}

La critique du naturalisme philosophique est en effet un thème présent au cœur de la phénoménologie husserlienne que ce soit dans La philosophie comme science rigoureuse ou les écrits de la Krisis. Cette critique va plus loin que le simple constat qu' "[e]n vertu de son point de départ toute science de la nature est naïve ${ }^{1}$ en tant qu'elle prend son objet comme donné sans interroger les modalités de cette donation même. Car le naturalisme ne se limite pas à cette seule "naïveté », il prétend en outre que seule l'analyse scientifique du monde physique nous donne l'heure juste sur la réalité : «Tout ce qui est, est soit physique et appartient comme tel à la totalité unifiée de la nature physique, soit psychique, mais alors il n'est qu'une simple variable dépendante du physique, tout au plus un fait secondaire qui l'accompagne parallèlement $»^{2}$.

On a dès lors affaire à une double opposition au projet phénoménologique. D'une part, l'entreprise fondationnelle de la phénoménologie consistant à montrer comment l'attitude des sciences de la nature doit nécessairement se fonder sur un travail philosophique préalable d'évaluation du rapport par lequel le monde se révèle à la conscience - est d'emblée jugée superflue par le naturalisme. C'est la profession de foi du naturaliste : la science donne accès à la réalité même. De ce fait, la phénoménologie husserlienne apparaît comme complètement superflue.

D'autre part, si la réalité du naturaliste se réduit au monde physique, à « [1]a nature comme le royaume des pures res extensae ${ }^{3}$ », et la conscience n'est plus qu'une unité «psychophysique » ${ }^{4}$, la phénoménologie se réduit alors à une province de la psychologie empirique. La «naturalisation de la conscience ${ }^{5}$ revient ainsi à dénaturer complètement l'intuition fondatrice de la phénoménologie husserlienne. D'une entreprise consistant à fonder l'attitude thétique du monde sur un examen approfondi de la conscience phénoménologique, on passerait, avec le naturalisme, à une analyse de la conscience qui présuppose a priori la validité de ce qu'initialement la phénoménologie husserlienne cherchait justement à établir.

\footnotetext{
${ }^{1}$ E. Husserl, La Philosophie comme science rigoureuse, Paris, PUF, 1955, p. 64.

${ }^{2}$ Ibid., p. 58.

3 E. Husserl, La Crise des sciences européennes et la phénoménologie transcendantale, Paris, Gallimard, 2012, p. 325.

${ }_{5}^{4}$ E. Husserl, La Philosophie comme science rigoureuse, op. cit., p. 58.

${ }^{5}$ Ibid., p. 62.
} 
On le voit, le naturalisme, tel que dépeint par Husserl, est antinomique avec son propre projet phénoménologique. Cela n'a toutefois pas empêché certains auteurs de suggérer récemment l'idée d'une "naturalisation de la phénoménologie », l'idée étant de «[lier] les descriptions husserliennes des phénomènes cognitifs et les sciences cognitives contemporaines $»^{1}$. Cela implique bien entendu un dépassement du tropisme antinaturaliste de la tradition phénoménologique. L'une des stratégies rhétoriques, pour ce faire, consiste à souligner à quel point la critique husserlienne du naturalisme était elle-même fondée sur des éléments contingents - tels le développement des sciences à son époque - aujourd'hui dépassés, rendant ainsi essentiellement obsolète sa position antinaturaliste ${ }^{2}$.

Il n'en reste pas moins que l'antinaturalisme husserlien exerça une influence durable sur l'ensemble du mouvement phénoménologique sans pour autant qu'on puisse simplement parler d'une reprise à l'identique des arguments husserliens. La Structure du comportement de Merleau-Ponty, par exemple, s'articule elle aussi autour d'une critique du naturalisme - en psychologie et en biologie essentiellement - sans pour autant que son entreprise soit entièrement tributaire de celle de Husserl. Pourrions-nous dès lors trouver de ce côté une critique du naturalisme qui échapperait aux critiques «contextualistes» des tenants d'une naturalisation de la phénoménologie consistant à dire que la validité des positions antinaturalistes de Husserl se limitait au contexte scientifique de son époque aujourd'hui dépassé ?

Encore faudrait-il savoir ce qu'on entend par «naturalisme». La définition donnée par Husserl et largement partagée par Merleau-Ponty consiste à faire du naturalisme une sorte de physicalisme réductionniste ${ }^{3}$. Toute la réalité se résume « en une multitude de processus partiels, extérieurs les uns aux autres [partes extra partes] dans le temps et l'espace ${ }^{4}$. Une telle conception du naturalisme a bien été défendue par le passé et l'est

\footnotetext{
1 J.-M. Roy, J. Petitot, B. Pachoud \& F. J. Varela. «Beyond the Gap : An Introduction to Naturalizing Phenomenology », in Id. (eds.) Naturalizing Phenomenology, Stanford, Stanford University Press, 1999, p. 43.

${ }^{2}$ Ibid., p. 40, 42-43. Notre travail s'articulant autour de la pensée de Merleau-Ponty, nous laisserons de côté la question de savoir si c'est le cas ou non que l'antinaturalisme husserlien soit effectivement dépassé historiquement autorisant ainsi des auteurs comme Roy, Petitot et al. à entreprendre le projet d'une naturalisation de la phénoménologie.

${ }^{3} \mathrm{SC}, \mathrm{p} .110-11$.

${ }^{4}$ Ibid., p. 7. Cf. H. Spiegelberg, The Phenomenological Movement, The Hague, Martinus Nijhoff, 1965, p. 539.
} 
certainement, pour une part, encore aujourd'hui. Quine, par exemple, dans «Epistemology Naturalized», réduit l'épistémologie à un chapitre de la psychologie qui n'est elle-même qu'un chapitre des sciences de la nature ${ }^{1}$. Mais ce n'est certainement pas là l'unique forme de naturalisme présente au sein des débats contemporains ${ }^{2}$.

Peter Godfrey-Smith défendra ainsi une version pour ainsi dire plus minimaliste du projet naturaliste en affirmant qu'un naturaliste tel qu'il l'entend admet simplement que la science peut contribuer par ses résultats aux questionnements philosophiques sans pour autant remplacer ces questions par des questionnements d'ordres proprement scientifiques ${ }^{3}$. Un tel naturalisme, qui admet l'apport légitime des recherches scientifiques au sein des débats philosophiques, nous apparaît plus conciliable avec certaines positions défendues par Merleau-Ponty. Celui-ci, en effet, fait constamment intervenir, principalement dans les deux premiers chapitres de La Structure $d u$ comportement, mais aussi dans la Phénoménologie de la perception, des résultats et travaux scientifiques pour appuyer son propos ou marquer ses critiques. Bien qu'il lui arrive de remettre en question les conclusions tirées par certains chercheurs à partir de ces résultats ${ }^{4}$, Merleau-Ponty ne doute pas de la légitimité qu'il puisse y avoir à évoquer de tels résultats dans le cadre d'une réflexion philosophique. Comme le souligne Spiegelberg dans son portrait du jeune Merleau-Ponty, celui-ci est dans un dialogue constant avec la science de son temps ${ }^{5}$. Par contre, alors que le naturalisme d'un GodfreySmith consisterait à dire que les résultats de la science peuvent apporter des réponses à la philosophie, Merleau-Ponty prétend, quant à lui, plutôt faire l'inverse, c'est-à-dire montrer en quoi la philosophie peut elle aussi apporter des réponses aux sciences dans l'impasse :

What is perhaps most characteristic of the early Merleau-Ponty is the concrete and painstaking manner in which he uses science as his point of departure and

\footnotetext{
${ }^{1}$ W. V. Quine, «Epistemology Naturalized », in E. Sosa \& J. Kim (eds.), Epistemology - An Anthology, op. cit., p. 297.

2 Sur quelques variantes contemporaines du naturalisme, $c f$. P. Poirier, «Unité et diversité du cognitivisme en théorie de la connaissance », in R. Nadeau (dir.), Philosophie de la connaissance, Québec, PUL/Vrin, 2009, p. 519-49.

3 «A naturalist can think that science can contribute to the answers to philosophical questions, without thinking that science should replace philosophical questions with scientific questions » (P. Godfrey-Smith, Theory and Reality, Chicago, The University of Chicago Press, 2003, p. 151).

${ }^{4} \mathrm{~L}$ 'exemple de Pavlov est à ce titre très parlant, $c f$. SC, p. 77-82.

${ }^{5}$ H. Spiegelberg, The Phenomenological Movement, op. cit., p. 540.
} 
works his way methodically to the place where only a new philosophical solution can do justice to the problem posed by it ${ }^{1}$.

On en trouve un bel exemple au commencement du chapitre III de $L a$ Structure du comportement où Merleau-Ponty souligne à quel point l'impasse de la Gestaltpsychologie est due, en définitive, à l'absence d'un travail approprié d'élucidation philosophique de la notion de « forme » :

La théorie de la forme est consciente des conséquences qu'entraîne une pensée purement structurale et cherche à se prolonger en une philosophie de la forme qui se substituerait à la philosophie des substances. Elle n'a jamais poussé très loin ce travail d'analyse philosophique. C'est que la « forme» ne peut être pleinement comprise et toutes les implications de cette notion dégagées que dans une philosophie qui s'affranchirait des postulats réalistes qui sont ceux de toute psychologie ${ }^{2}$.

En l'absence d'un tel travail d'analyse philosophique, la psychologie de la forme retombe elle-même dans les vieux réflexes « réalistes » et aspire à une «naturalisation de la forme ${ }^{3}$ » consistant à faire de la structure ou de la forme une chose du monde. On assiste alors à un repli de l'ordre cognitif sur l'ordre naturel ou physique sans être en mesure d'expliciter la nature même d'une telle correspondance. Cette incapacité, la Gestalttheorie, du fait de son manque d'élucidation philosophique, la partage, aux yeux de Merleau-Ponty, avec le mécanisme et le béhaviorisme. On retombe alors du côté de l'épistémologie médiationnelle décriée par Dreyfus et Taylor. En tentant de montrer comment la forme comme chose du monde peut causer une représentation dans la conscience, la Gestalttheorie propose une harmonie entre l'ordre mental et l'ordre naturel qu'il s'agit justement d'expliquer et non simplement de poser.

D'ailleurs, une telle "harmonie » suggère d'emblée une séparation, une dualité initiale. S'il faut « harmoniser » le psychique et le physique, c'est qu'au départ ils constituent deux domaines distincts de la réalité. L'approche de Merleau-Ponty, pour sortir de l'impasse classique du dualisme médiationnel, sera, comme nous l'avons montré, tout autre. Plutôt que de prendre le problème en aval, la solution de Merleau-Ponty, en continuité avec, bien qu'à distinguer de celle de Husserl, prend le problème en amont.

${ }^{1} I d$.

${ }^{2}$ SC, p. 200.

${ }^{3}$ Ibid., p. 203. 
Le naturalisme, tout comme l'intellectualisme, part en effet des deux termes en oppositions - l'esprit et le monde - pour réduire l'un à l'autre. Les deux approches sont ainsi essentiellement réductionnistes. Mais si une telle réduction s'avère impossible à compléter - et il y a bien des raisons pour le penser, selon Merleau-Ponty - nous nous retrouvons alors à la case départ, à la recherche d'une nouvelle manière pour comprendre le rapport entre l'esprit et le monde. Ce qui caractérise donc plus particulièrement la critique merleau-pontienne du naturalisme, c'est son rejet de tout réductionnisme ${ }^{1}$. En ce sens, Taylor s'inscrit parfaitement dans la lignée de Merleau-Ponty lorsqu'il écrit qu'en définitive l'ambition réductionniste est une pure fantaisie ${ }^{2}$.

Il faut d'ailleurs souligner que, pour quelqu'un comme Rorty, la critique de Merleau-Ponty à l'endroit du naturalisme se résume essentiellement à cette critique du réductionnisme, de sorte qu'on peut très bien adopter une position naturaliste tout en étant pour l'essentiel en accord avec Merleau-Ponty (ce que prétend d'ailleurs faire Rorty) :

I see myself as a wholehearted naturalist, but one who is as antireductionist as Taylor himself [...]. I regard myself as cleansed of reductionnist sin. But this redemption makes me all the more eager to insist, against Taylor, that Heidegger and Merleau-Ponty do not show us how to avoid naturalism, but merely caution us against letting naturalism slide over into reductionism ${ }^{3}$.

Il est vrai que l'essentiel de la critique merleau-pontienne du naturalisme tourne autour d'une critique réductionniste - ce qui fait d'ailleurs que cette critique présente certains motifs similaires à la critique de l'intellectualisme à l'œuvre dans la Phénoménologie de la perception -, mais cela ne veut pas dire pour autant que Merleau-Ponty puisse être réconcilié aussi facilement que le prétend Rorty avec une position naturaliste. Une telle position pose le plus souvent la nature uniquement en termes de causalité physique ${ }^{4}$, et ce, même lorsqu'elle n'est pas à proprement parler réductionniste, c.-à-d. qu'elle ne réduit pas de facto le monde de la signification et des normes - la sphère pratique - à sa simple expression physico-chimique. Or, pour Merleau-

\footnotetext{
${ }^{1}$ « Merleau-Ponty rejects any form of reductionism or eliminativism - the view he would call "naturalism" " (H. Dreyfus, "Merleau-Ponty and Recent Cognitive Science », art. cit., p. 142).

2 « The reductive ambition is ultimately a fantasy » (Ch. Taylor, « Foundationalism and the Inner-Outer Distinction », art. cit., p. 111).

${ }^{3}$ R. Rorty, « Charles Taylor on Truth », art. cit., p. 94-95.

${ }^{4}$ Ibid., p. 94.
} 
Ponty, une telle « réduction » de la nature à sa réalité physique, même si elle fait place, dans l'ordre du réel, aux sphères pratique et sociale, à la « seconde nature » de l'être humain, demeure déficiente. La nature n'est pas que le règne de la causalité, celle-ci n'étant qu'une forme dialectique parmi d'autres, celle qui relève, pour Merleau-Ponty de l'ordre physique de la nature, alors que l'ordre vital, tout aussi naturel, présente les rapports dialectiques de l'animal vivant et son milieu en des termes qui sont, à ses yeux, irréductibles à l'ordre causal ${ }^{1}$. C'est pourquoi, si l'on veut, comme certains ont tenté de le faire ${ }^{2}$, défendre une sorte de naturalisme merleaupontien, il faut non seulement admettre au-delà de la nature une sphère pratique irréductible à l'ordre causal du monde, il faut en outre dépeindre la nature elle-même comme étant irréductible à l'ordre causal (physique). La causalité, valide dans l'ordre physique ne peut, selon Merleau-Ponty, convenir à la description de la nature selon l'ordre vital.

Il faut dès lors affirmer que le rapport qu'entretient Merleau-Ponty avec le naturalisme est certainement plus ambivalent - et, à ce titre, caractéristique de cette "philosophie de l'ambiguïté »" qui, selon l'expression de de Waelhens, définit si bien la pensée merleau-pontienne - que ne le suggèrent souvent les tenants du naturalisme désireux de se réapproprier sa pensée. Il également plus ambivalent que le rapport très critique de Husserl.

\section{Merleau-Ponty et le Mythe du donné}

De fait, nous avons vu que la critique husserlienne du naturalisme partait, dans une certaine mesure, d'un point de vue fondationaliste. Si le naturalisme est à repousser, c'est qu'il prétend rendre compte de la réalité même sans entreprendre le travail préalable d'une explicitation phénoménologique des conditions par lesquelles les choses du monde se donnent comme objets à la conscience. La phénoménologie se présente ainsi comme le travail d'exposer les fondations sur lesquelles s'appuie tout rapport thétique au monde.

Dans la mesure où le fondationalisme constitue, pour Dreyfus et Taylor, avec le représentationalisme, l'un des éléments de l'épistémologie médiationnelle à dépasser absolument, et dans la mesure où Merleau-Ponty

${ }^{1}$ Cf. T. Geraets, Vers une nouvelle philosophie transcendantale, op. cit., p. 40-41, 65.

${ }^{2} C f$., par exemple, R. G. Brice \& P. L. Bourgeois, « Naturalism Reconsidered Wittgenstein and Merleau-Ponty », Philosophy Today, vol. 56, no. 1, 2012, p. 78-83.

${ }^{3}$ A. de Waelhens, « Une philosophie de l'ambiguïté », in SC, p. V. 
demeure, dans une large mesure, marqué par ce tropisme antinaturalisme de la phénoménologie allemande, il importe alors de déterminer si l'alternative merleau-pontienne à l'épistémologie médiationnelle - qu'elle soit naturaliste, intellectualiste ou dualiste - ne retombe pas elle aussi dans une forme de fondationalisme en lui-même problématique.

En cherchant à montrer comment l'attitude du spectateur étranger et objectif, de l'enfant — pour maintenir l'analogie — jouant le jeu du savoir est, en définitive, qu'une forme dérivée de la conscience naïve, MerleauPonty ne propose-t-il pas un nouveau fondement au savoir? Ne présente-t-il pas une forme d'épistémologie fondationaliste posant la possibilité du savoir scientifique sur le socle solide de notre être-au-monde?

Le fondationalisme, c.-à-d. cette volonté de dépouiller (stripping down) les couches de sens jusqu'à un donné préconceptuel et incontestable, constitue cependant, pour Taylor, non pas qu'un effet secondaire de l'épistémologie médiationnelle. Il en est l'un des fondements ${ }^{1}$. Le dépassement de l'épistémologie médiationnelle aurait ainsi pour condition nécessaire l'abandon de tout fondationalisme. On peut dès lors difficilement envisager que l'abandon de l'épistémologie médiationnelle prenne au final la forme d'un nouveau fondationalisme. C'est d'ailleurs manifestement contre toute forme de fondationalisme que Dreyfus et Taylor se revendiquent de la critique sellarsienne du mythe du donné ${ }^{2}$. Mais il faut alors se demander si leur approche «contactiste» plutôt que médiationnelle n'offre pas, comme le suggère Berendzen pour Dreyfus ${ }^{3}$, elle-même une nouvelle version de ce mythe.

Certes, comme l'a très bien montré Soffer dans son article sur Husserl et Sellars, la phénoménologie - husserlienne à tout le moins - échappe à la forme classique du mythe du donné en rejetant la prétention thétique du donné sensible. Pour la phénoménologie comme pour Sellars, le sense-datum n'a rien d'une évidence qui se donne soi-même (self-evidence) et s'inscrit plutôt dans un réseau de jugements implicites (concernant l'arrière-plan de l'apparaître du donné, le caractère commun ou intersubjectif du donné, etc.). Ces jugements ne requièrent cependant pas, pour Husserl, contrairement à Sellars, une maîtrise réflexive du langage conceptuel. La conscience naïve, l'enfant, voire l'animal, perçoivent le donné à partir d'une articulation de

\footnotetext{
${ }^{1} \mathrm{Ch}$. Taylor, « Foundationalism and the Inner-Outer Distinction », art. cit., p. 109.

${ }^{2}$ H. Dreyfus \& Ch. Taylor, Retriving Realism, op. cit., p. 28.

${ }^{3}$ Voir n. 1.
} 
sens qui n'a pas de facto besoin de prendre une forme élaborée, conceptuelle et linguistique ${ }^{1}$.

Que Husserl échappe au piège du mythe du donné dans sa forme primitive n'exclut pas toutefois qu'il ne puisse offrir au final qu'une nouvelle version plus sophistiquée du même mythe. Pour Sellars, en effet, le mythe du donné est une véritable hydre: lorsqu'on lui coupe la tête, de nouvelles versions réapparaissent aussitôt ${ }^{2}$. D'ailleurs, derrière la critique du mythe du donné, c'est avant tout une critique de l'immédiateté qu'articule Sellars. Dès le $\S 1$ d'Empirisme et philosophie de l'esprit, celui-ci suggère en effet l'équivalence conceptuelle entre "l'idée philosophique de donné [givenness $] »$ et celle «d'immédiateté $»^{3}$. «[E]u égard au donné épistémologique », la sphère du vécu n'offre ainsi «ni aide ni soutien ${ }^{4}$. Notre savoir s'appuie toujours déjà sur un ensemble de concepts ainsi que les méthodes permettant d'articuler ces concepts en termes de raisons et de justification. La seule sortie définitive du donné doit ainsi s'inscrire, selon Sellars, dans le langage en tant qu'il est la précondition à l'articulation de nos concepts, ceux-ci étant eux-mêmes nécessairement mobilisés dans notre expérience quotidienne du monde ${ }^{5}$. L'approche de Sellars s'oppose ainsi à ce qui constitue au contraire, pour Merleau-Ponty, notre véritable expérience du monde, dans sa forme engagée (engaged) ${ }^{6}$ et adaptée - ce que Dreyfus nomme l' « ordinary coping ».

${ }^{1} C f$. G. Soffer, « Revisiting the Myth : Husserl and Sellars on the Given », art. cit., p. $308-10$.

2 « Husserl is not committed to the Myth of the Given in its basic form. However, for Sellars the Myth is a veritable Hydra, turning up from classical empiricism to logical positivism, from Descartes to Hegel » (ibid., p. 310-11). Cf. W. Sellars, Empirisme et philosophie de l'esprit, op. cit., p. 17-18, § 1.

${ }^{3}$ Ibid., p. 17, § 1 .

${ }^{4}$ Ibid., p. 82, § 39.

${ }^{5}$ Cf. ibid., p. 91, § 45 ; G. Soffer, « Revisiting the Myth : Husserl and Sellars on the Given », art. cit., p. 311. C'est, en définitive, en montrant que cette idée sellarsienne - que seul le tournant linguisitique (linguistic turn) peut échapper effectivement et définitivement au mythe du donné - est fausse — à la lumière des développements des sciences cognitives et de l'abandon du programme béhavioriste sur lequel s'appuyait Sellars - que Soffer entend démontrer que Husserl ne tombe pas à nouveau dans le mythe du donné, malgré l'articulation de sa philosophie autour du vécu subjectif immanent à la conscience. La position de Soffer ne peut malheureusement pas faire l'objet d'un commentaire critique dans le présent texte.

${ }^{6}$ H. Dreyfus \& Ch. Taylor, Retriving Realism, op. cit., p. 36. 
Alors que, pour Sellars, notre structure perceptive ne se comprend pleinement qu'à partir du moment où nous en saisissons la dimension conceptuelle sous-jacente (à la manière de Kant), pour Merleau-Ponty, au contraire, c'est cette structure perceptive originaire qui constitue le véritable fondement à partir duquel un mode "désengagé », objectif et neutre - celui du spectateur-expert ou du scientifique - peut émerger ${ }^{1}$. Or, cette structure originaire de notre être-au-monde est essentiellement préconceptuelle, prélangagière : «Ordinary coping isn't conceptual ${ }^{2}$. Ce n'est pas à partir du moment où l'on en comprend le sous-bassement conceptuel et linguistique qu'on échappe à l'impasse des épistémologies médiationnelles, c'est, au contraire, en comprenant comment concept et langage dérivent tous deux d'une telle expérience originaire :

C'est sur l'expérience du monde que doivent se fonder toutes nos opérations logiques de signification, et le monde lui-même n'est donc pas une certaine signification commune à toutes nos expériences que nous lirions à travers elles, une idée qui viendrait animer la matière de la connaissance ${ }^{3}$.

C'est parce que nous sommes immédiatement «au-monde» comme sujet incarné et percevant que nous pouvons connaître le monde - autant selon le mode de l'adaptation ordinaire (ordinary coping) que celui de la science. La «fonction essentielle» de la perception, selon Merleau-Ponty, est «de fonder ou d'inaugurer la connaissance ${ }^{4}$. Percevoir est notre manière d'êtreau-monde. Au travers de la perception, la conscience naïve accède directement au monde, à sa réalité ${ }^{5}$. Comme corps situé, le sujet interagit

\footnotetext{
${ }^{1}$ PP, p. 494.

${ }^{2}$ H. Dreyfus \& Ch. Taylor, Retriving Realism, op. cit., p. 51.

${ }^{3}$ PP, p. 379.

${ }^{4}$ Ibid., p. 24.

${ }^{5}$ Il semble en effet que, pour Merleau-Ponty, la perception possède en définitive une certaine valeur thétique qui échappe toujours minimalement à la réduction phénoménologique ( $c f$. PP, p. VIII, 452 ; SC, p. 284-85). Ce n'est qu'à un niveau supérieur de réflexivité que l'on accède à une forme de perception non thétique telle qu'elle se présente chez Husserl (PP, p. 61). Il faut en effet attendre que la consience naïve devienne conscience de soi, capable d'introspection et de réflexivité pour mettre entre parenthèses la valeur thétique du monde. En ce sens, l'attitude phénoménologique, tout comme l'attitude scientifique, constitue une forme dérivée de l'expérience naïve du monde. Elle se démarque cependant de l'attitude scientifique par sa volonté d'expliciter non seulement la structure originaire de l'être-au-monde de la conscience naïve, mais aussi ses formes dérivées que constituent l'attitude scientifique et phénoménologique. Ce faisant, cependant, la phénoménologie, con-
} 
immédiatement avec le monde des choses et autrui ${ }^{1}$. Mais ce faisant, ne sommes-nous pas retombés dans une forme nouvelle de fondationalisme? Si ce n'est plus le cogito ou les sense-data qui fondent la connaissance du monde, n'est-ce pas la structure même de notre être-au-monde comme sujet incarné et percevant?

Si tel est le cas, l'approche " contactiste » de Dreyfus et Taylor se retrouve face à une difficulté. D'un côté, elle rejette toute forme de fondationalisme comme étant l'un des deux éléments constitutifs de l'épistémologie médiationnelle, de l'autre côté, elle semble elle-même s'appuyer sur l'être-au-monde merleau-pontien comme sur nouveau fondement de tout savoir. C'est pourquoi il est primordial de déterminer si la position merleaupontienne constitue bel et bien, en définitive, une nouvelle version du mythe du donné ou si, au contraire, elle réussit à y échapper.

Comme le suggère Carl B. Sachs ${ }^{2}$, par certains passages, MerleauPonty, semble en effet se référer au donné en son sens «pernicieux et mythique » critiqué par Sellars. Il en donne pour exemple le passage suivant :

Le monde naturel est l'horizon de tous les horizons, le style de tous les styles, qui garantit à mes expériences une unité donnée et non voulue par-dessous toutes les ruptures de ma vie personnelle et historique, et dont le corrélatif est en moi l'existence donnée, générale et prépersonnelle de mes fonctions sensorielles où nous avons trouvé la définition du corps ${ }^{3}$.

trairement à la science, aspire à rendre compte de ses propres conditions de possibilité. C'est tout l'enjeu d'une « phénoménologie de la phénoménologie » (PP, p. 419), à savoir faire une description phénoménologique de l'attitude phénoménologique même, tout comme Merleau-Ponty cherche à faire une description phénoménologique de l'attitude scientifique en partant de la conscience naïve. Mais dans la mesure où la perception originaire est dans une certaine mesure thétique, la critique sellarsienne du donné retrouve une certaine force face la Merleau-Ponty qu'elle n'avait pas face à Husserl (contrairement à ce qui est implicitement suggéré par Sachs, $c f$. C. B. Sachs, "Discursive and Somatic Intentionality: Merleau-Ponty Contra 'McDowell or Sellars' ", International Journal of Philosophical Studies, vol. 22 , no.2, 2014, p. 210-11).

${ }^{1}$ Sur l'évidence de la présence pleine et entière d'autres consciences et le dépassement du problème classique de l'intersubjectivité chez Merleau-Ponty, $c f$. SC, p. $261 ;$ PP, p. 403, 407.

${ }^{2}$ C. B. Sachs, « Discursive and Somatic Intentionality... », art. cit., p. 210.

${ }^{3}$ PP, p. 381 [nous soulignons]. 
Le monde se donne à soi dans son évidence, non pas en termes de sensations comme le pensait l'empiriste, mais en termes de perceptions. Dans les deux cas cependant, la dimension conceptuelle n'apparaît qu'ensuite :

Quand je perçois une chose [...] ce n'est pas la concordance de ses différents aspects qui me fait conclure à l'existence de la cheminée comme géométral et signification commune de toutes ces perspectives, mais inversement je perçois la chose dans son évidence propre et c'est ce qui me donne l'assurance d'en obtenir, par le déroulement de l'expérience perceptive, une série indéfinie de vues concordantes ${ }^{1}$.

Le mythe du donné sensitif n'a-t-il pas été simplement remplacé par le mythe du donné perceptif? On retrouve en effet, chez Merleau-Ponty une critique quasi-sellarsienne de la sensation comme donné dès les premières pages de la Phénoménologie de la perception ${ }^{2}$. Pour Merleau-Ponty aussi, la sensation n'est jamais un donné, mais le produit dérivé d'une articulation toujours déjà conceptuelle $^{3}$. Cependant, plutôt que de poser conséquemment la connaissance comme le fruit d'une articulation conceptuelle dans l'espace logique des raisons, Merleau-Ponty fait d'une certaine manière le geste inverse, en cherchant à déterminer une structure plus originaire que celle de la sensation à partir de laquelle il serait possible de penser notre rapport immédiat - et préconceptuel - au monde. Mais c'est l'idée même d'une telle structure préconceptuelle et prélinguistique qui était l'objet sous-jacent de la critique sellarsienne de l'empirisme. Comment, dès lors, Merleau-Ponty échappe-t-il à une nouvelle version du mythe ?

Si Merleau-Ponty affirmait simplement qu'un retour à la structure originaire de la perception constituait la condition nécessaire et suffisante de notre savoir, il succomberait effectivement, de manière générale, au mythe du donné. Mais tel n'est pas le cas : «La synthèse du monde perçu n'est pas un pur donné, une chose passivement reçue ${ }^{4}$. La perception se rapporte toujours à un réseau de perceptions mises en relations les unes aux autres. Le

\footnotetext{
${ }^{1}$ Ibid., p. 216.

${ }^{2}$ PP, p. 9.

${ }^{3}$ « La théorie de la sensation, qui compose tout savoir de qualités déterminées, nous construit des objets nettoyés de toute équivoque, purs, absolus, qui sont plutôt l'idéal de la connaissance que ses thèmes effectifs, elle ne s'adapte qu'à la superstructure tardive de la conscience » (ibid., p. 18-19). Cf. ibid., p. 17, 279.

${ }^{4}$ Ibid., p. 276.
} 
perçu n'est jamais un donné autonome, indépendant, il s'inscrit dans la cohérence d'un réseau de sens vécu ${ }^{1}$.

Mais l'unité de l'expérience perceptive n'est pas davantage le produit de l'activité d'une conscience transcendantale ou du cogito cartésien. On pourrait en effet croire que, si Merleau-Ponty échappe à la version empiriste du donné en posant la perception dans un réseau de sens, bien que celui-ci soit originairement préconceptuel, il succombe néanmoins à la version intellectualiste du donné. Selon cette version, c'est le cogito qui constitue le fondement, immédiatement donné à soi — bien que le plus souvent recouvert par les couches de sens héritées de la tradition - du savoir. Une fois la conscience donnée ou dévoilée, l'intellectualiste prétend en effet reconstituer à partir de celle-ci un savoir plein et entier du monde. Une telle possibilité est cependant explicitement refusée par Merleau-Ponty. Qu'elle soit transcendantale, cartésienne ou incarnée, la conscience n'a jamais qu'une vue partielle sur le monde : «Elle ne constitue pas le monde, elle le devine autour d'elle comme un champ qu'elle ne s'est pas donné $)^{2}$. Si l'être-au-monde constitue bien une condition à notre savoir du monde, il n'en constitue pas cependant une condition suffisante. Pour Merleau-Ponty tant l'illusion que le savoir ne peuvent être absolus ${ }^{3}$. L'exclusion du scepticisme n'implique aucun triomphalisme de la raison. Et c'est pourquoi la version intellectualiste du mythe du donné reste étrangère à la position merleau-pontienne.

Mais il y a encore une raison supplémentaire pour laquelle l'être-aumonde merleau-pontien n'est pas une nouvelle forme du mythe du donné. Cette raison est que la structure même de l'être-au-monde appelle une vaste entreprise d'explicitation. Que signifie précisément être-au-monde comme corps percevant? La Phénoménologie de la perception doit justement se lire comme un vaste travail d'explicitation phénoménologique de cette structure de notre être-au-monde comme conscience percevante.

Ce qui constitue le fondement originaire de notre connaissance n'a donc rien d'évident en soi, il n'est en aucun cas donné, transparent à soi, mais doit au contraire être analysé dans le détail. Une telle analyse, pour

\footnotetext{
${ }^{1}$ «Une première perception sans aucun fond est inconcevable. Toute perception suppose un certain passé du sujet qui perçoit et la fonction abstraite de la perception, comme rencontre des objets » (ibid., p. 326). Cf. également ibid., p. 377.

${ }^{2}$ PP, p. 462. «L'expérience n'est pas déployée devant moi comme si j'étais Dieu, elle est vécu par moi d'un certain point de vue, je n'en suis pas le spectateur, j'y suis partie, et c'est mon inhérence à un point de vue qui rend possible à la fois la finitude de ma perception et son ouverture au monde total comme horizon de toute perception » (ibid., p. 350 ; voir également ibid., p. 399).

${ }^{3}$ Ibid., p. 396.
} 
Merleau-Ponty, n'exclut d'ailleurs pas totalement l'apport des recherches issues des sciences empiriques. De fait, si notre être-au-monde est originaire et que l'attitude scientifique est dérivée de celui-ci, l'explicitation de cet êtreau-monde exige néanmoins des formes plus élaborées de discours que ce qui constitue originairement l'attitude de la conscience naïve. L'explicitation phénoménologique de l'être-au-monde originaire présuppose d'emblée cette distanciation même du spectateur dont elle cherche à expliquer l'origine ${ }^{1}$. Une telle circularité n'a cependant rien de vicieux. Certes, la conscience réflexive présuppose d'un point de vue existentiel la conscience préréflexive qu'elle cherche à atteindre dans sa description phénoménologique, mais par le biais de l'épochè, la conscience phénoménologique atteint cette conscience originaire, transcendantale, préréflexive, sans en présupposer a priori l'existence. C'est ainsi que, d'un point de vue phénoménologique, elle la découvre sans la présupposer, bien que l'entreprise phénoménologique ellemême, d'un point de vue existentiel, constitue, pour Merleau-Ponty, une figure effective de la conscience dérivée par rapport à cet être-au-monde originaire.

Par ailleurs, en soulignant la nécessité d'une telle entreprise d'explicitation de la perception comme structure de notre être-au-monde, MerleauPonty montre implicitement que la phénoménologie comme description s'inscrit aussi dans l'espace des raisons, du langage et de la conceptualité bien que son objet puisse, quant à lui, échapper à l'espace des raisons pour en constituer le fondement même. Certes, pour Merleau-Ponty, la conscience naïve est prélinguistique et préconceptuelle, mais pour en faire la description et l'explicitation, nous devons nous situer du côté du langage et du concept ${ }^{2}$. Et l'explicitation phénoménologique de notre être-au-monde peut être plus ou moins bien menée, devenant ainsi objet de débat (sur la méthode, l'appareil conceptuel, etc.). C'est en ce sens que l'entreprise merleaupontienne échappe également au donné, en s'inscrivant, comme discours, comme conceptualisation explicitante de notre être-au-monde, au sein d'un espace des raisons où elle continue à faire l'objet de critiques et de défenses. À la différence près qu'elle affirme qu'un tel espace des raisons, un tel jeu du savoir constitue néanmoins toujours une forme de vie dérivée de l'être-aumonde originaire. En prenant position au sein de l'espace des raisons, Merleau-Ponty, avec Dreyfus et Taylor à sa suite, ne défend pas seulement une position parmi d'autres, mais prétend montrer comment un tel espace en vient justement à pouvoir exister. Contrairement à ce qu'en dit Brandom,

${ }^{1}$ C. B. Sachs, « Discursive and Somatic Intentionality... », art. cit., p. 212.

${ }^{2}$ PP, p. 388. 
prendre position au sein de l'espace des raisons (standing in the space of reason) n'est pas qu'une question de rang (standing) ou de statut ${ }^{1}$, c'est aussi une question de perspectives, c.-à-d. de la manière dont je prends position comme corps percevant ${ }^{2}$. Ce n'est pas seulement mon horizon spatial, mon champ visuel qui dépend de la position de mon corps dans l'espace, c'est tout mon horizon conceptuel, mon champ épistémique qui est déterminé par mon expérience comme conscience incarnée au-monde ${ }^{3}$.

\section{Conclusion}

Comme j'ai tenté de le montrer, l'approche «contactiste » de Dreyfus et Taylor présente donc, pour les réflexions épistémologiques désireuses de dépasser l'impasse du cadre médiationnel, un terreau fertile. Il l'est encore davantage lorsqu'on s'attarde attentivement aux textes de Merleau-Ponty qui en constituent l'une des sources les plus marquantes. En effet, bien des aspects de la critique de Dreyfus et Taylor sont déjà anticipés par MerleauPonty, qui présente en outre une critique originale des perspectives naturaliste-réaliste et intellectualiste.

La force d'une position critique comme celle de Dreyfus, Taylor et Merleau-Ponty ne se mesure cependant qu'à sa capacité à interroger ses propres présupposés. C'est ce que j'ai tenté de faire en confrontant l'être-aumonde merleau-pontien à la critique sellarsienne du donné. J'ai ainsi montré en quoi l'être-au-monde ne pouvait être accusé de succomber au mythe du donné dans la mesure où l'articulation de notre connaissance, si elle se fait effectivement à partir de notre structure perceptive originaire, ne se concrétise que progressivement et n'est jamais donnée comme tel. Il reste cependant à clarifier plus avant la signification d'une telle conclusion, car si la théorie-contact dans sa version merleau-pontienne échappe au mythe du donné, c'est notamment parce qu'en tant que discours sur la structure préréflexive de la conscience naïve percevante, elle relève aussi d'un espace conceptuel comme l'espace des raisons où elle est sujette à discussion. Or, cela revient-il à réintroduire une sorte de séparation entre l'être-au-monde comme tel et notre compréhension représentative de cet être-au-monde au sein de l'espace des raisons? Comme nous l'avons brièvement esquissé en

\footnotetext{
${ }^{1}$ R. Brandom, «Knowledge and the Social Articulation of the Space of Reasons », art. cit., p. 428.

${ }^{2}$ SC, p. $282-84$.

${ }^{3}$ PP, p. 236.
} 
toute fin de la section précédente, s'il l'on peut répondre par la négative à cette question et ainsi sauver le « contactisme » d'un nouveau dualisme, c'est dans la mesure où il faut penser l'espace des raisons lui-même comme constitué, non pas par des consciences désincarnées, mais bien par des consciences «au-monde», capables d'adopter la position d'un spectateur objectif et détaché, mais toujours en se distanciant par rapport à leur êtreimmédiat et originaire, au-monde. Il faudrait cependant, au minimum, un texte à part entière consacré à l'explicitation d'une telle réforme merleaupontienne de l'espace sellarsien des raisons. Un tel texte, s'il vient à être, nécessitait cependant lui-même, au préalable, le travail d'éclaircissement conceptuel que nous avons entrepris ici.

\section{Bibliographie}

Augustin, Confessions, Paris, Pierre Horay, 1961.

Berendzen J. C., «Coping Without Foundations: On Dreyfus's Use of MerleauPonty », International Journal of Philosophical Studies, vol. 18, no. 5, 2010, p. 629-649.

Brandom Robert, «Knowledge and the Social Articulation of the Space of Reasons », in Ernest Sosa \& Jaegwon Kim (eds.), Epistemology - An Anthology, London, Blackwell, 2005, p. 424-432.

Brice Robert G. \& Bourgeois Patrick L., « Naturalism Reconsidered - Wittgenstein and Merleau-Ponty », Philosophy Today, vol. 56, no. 1, 2012, p. 78-83.

Descombes Vincent, Le Même et l'autre - Quanrante-cinq ans de philosophie française (1933-1978), Paris, Minuit, 1979.

De Waelhens Alphonse, «Une philosophie de l'ambiguïté », dans Maurice MerleauPonty, La Structure du comportement, Paris, PUF, 2013, p. v-xviii.

Dreyfus Hubert, « Merleau-Ponty and Recent Cognitive Science », in Taylor Carman and Mark B. N. Hansen (eds.), The Cambridge Companion to Merleau-Ponty, Cambridge, Cambridge University Press, 2006, p. 129-150.

Dreyfus Hubert \& Taylor Charles, Retriving Realism, Cambridge, Harvard University Press, 2015.

Geraets Theodore F., Vers une nouvelle philosophie transcendantale - La genèse de la philosophie de Maurice Merleau-Ponty jusqu'à la Phénoménologie de la Perception, La Haye, Martinus Nijhoff, 1971.

Godfrey-Smith Peter, Theory and Reality, Chicago, The University of Chicago Press, 2003.

Husserl Edmund, La Philosophie comme science rigoureuse, Paris, PUF, 1955.

Husserl Edmund, La Crise des sciences européennes et la phénoménologie transcendantale, Paris, Gallimard, 2012. 
Merleau-Ponty Maurice, « Le Cinéma et la nouvelle psychologie », dans Sens et nonsens, Paris, Nagel, 1965, p. 85-106.

Merleau-Ponty Maurice, Phénoménologie de la perception, Paris, Gallimard, 1967.

Merleau-Ponty Maurice, La Structure du comportement, Paris, PUF, 2013.

Poirier Pierre, "Unité et diversité du cognitivisme en théorie de la connaissance », dans Robert Nadeau (dir.), Philosophie de la connaissance, Québec, PUL/Vrin, 2009, p. 519-550.

Quine W. V., «Epistemology Naturalized», in Ernest Sosa \& Jaegwon Kim (ed.), Epistemology - An Anthology, London, Blackwell, 2005, p. 292-300.

Rorty Richard, "Charles Taylor on Truth", in Truth and Progress - Philosophical Papers, Volume 3, Cambridge, Cambridge University Press,1998, p. 84-97.

Roy Jean-Michel, Petitot Jean, Pachoud Bernard \& Varela Francisco J., «Beyond the Gap: An Introduction to Naturalizing Phenomenology », in Jean Petitot, Francisco J. Varela, Bernard Pachoud, Jean-Michel Roy (eds.), Naturalizing Phenomenology, Stanford, Stanford University Press, 1999, p. 1-80.

Sachs Carl B., "Discursive and Somatic Intentionality: Merleau-Ponty Contra "McDowell or Sellars" ", International Journal of Philosophical Studies, vol. 22, no. 2, 2014, p. 199-227.

Sellars Wilfrid, Empirisme et philosophie de l'esprit, Paris, L'Éclat, 1992.

Sellars Wilfrid, "La Philosophie et l'image scientifique de l'homme», dans D. Fisette et P. Poirier (éds.), Philosophie de l'esprit-Psychologie du sens commun et sciences de l'esprit, Paris, Vrin, 2002, p. 55-115.

Soffer Gail, «Revisiting the Myth : Husserl and Sellars on the Given », The Review of Metaphysics, vol. 57, no. 2, 2003, p. 301-337.

Spiegelberg Herbert, The Phenomenological Movement, The Hague, Martinus Nijhoff, 1965.

Taylor Charles, « Foundationalism and the Inner-Outer Distinction », in Nicholas H. Smith (ed.), Reading McDowell on Mind and World, London, Routledge, 2002, p. 106-119.

Taylor Charles, "Merleau-Ponty and the Epistemological Picture», in Taylor Carman and Mark B. N. Hansen (eds.), The Cambridge Companion to MerleauPonty, Cambridge, Cambridge University Press, 2006, p. 26-49.

Wittgenstein Ludwig, Tractatus logico-philosophicus suivi des Investigations philosophiques, Paris, Gallimard, 1986. 